Classification

Physics Abstracts

$12.35 \mathrm{C}$

\title{
Chiral logarithms in quenched QCD
}

\author{
A. Morel \\ Service de Physique Théorique, CEN-Saclay, 91191 Gif-sur-Yvette Cedex, France
}

(Reçu le 16 février 1987, accepté le 27 mars 1987)

\begin{abstract}
Résumé. - A partir d'une étude de la chromodynamique quantique avec fermions gelés à couplage fort, et de l'analyse de résultats numériques pour le groupe $\mathrm{SU}(2)$, on montre que les logarithmes infrarouges attendus à la limite chirale ne sont pas supprimés en même temps que les boucles internes de quarks. Ce résultat explique la non-annulation apparente de l'extrapolation chirale de la masse du pion, antérieurement observée dans des simulations numériques. Il a des conséquences pratiques sur la détermination précise de la masse physique des quarks à partir du réseau et rend souhaitable une meilleure compréhension de la nature de l'approximation gelée.
\end{abstract}

\begin{abstract}
From a strong coupling investigation of quenched QCD, and an analysis of a previous numerical simulation of quenched SU(2), we show that the infrared logarithms expected in the chiral limit of complete QCD are not suppressed by the suppression of all internal quark loops. This result explains the apparent nonvanishing of $\pi$-mass extrapolations at zero quark mass, previously observed in several numerical studies. It has practical consequences for the precise determination from the lattice of physical quark masses. It calls for a better understanding of the nature of quenched QCD.
\end{abstract}

\section{Introduction.}

A consequence of spontaneous symmetry breaking in QCD is the appearance of Goldstone bosons in the chiral limit where the quark masses $m$ go to zero. Close to this limit, infrared logarithmic singularities are anticipated in physical quantities, associated with the large distance fluctuations implied by the (quasi) zero modes [1]. Precise evaluations of these chiral logarithms have been obtained, leading to the result that the corrections to the $m=0$ limit are not of order $m$, but rather of order $m \log m$. A complete investigation of this issue has been performed by Gasser and Leutwyler [2,3], whose papers contain a list of references to earlier and related works. It is found that the coefficient of $\ln (m)$ is proportional to the ratio $M_{0}^{2} / F_{0}^{2}$ of the squared mass $\left(M_{0}^{2} \sim s m\right)$ to the squared decay constant of the pion (throughout this paper, we disregard flavour breaking effects ; there is only one quark mass $m$, and all flavour nonsinglet Goldstone pseudo-scalars are generically called pions). It is argued in the above quoted works that the effect of these corrections can be substantial.

Chiral symmetry breaking and the associated phenomena just outlined are non-perturbative effects in QCD, which numerical simulations with a lattice regularization ought to reproduce in a quantitative manner. There are indeed good evidences that chiral symmetry is spontaneously broken, based both on analytical (strong coupling) and numerical studies. Using the Kogut-Susskind regularization of fermions, which preserves part of the chiral symmetry of a 4-flavour continuum QCD, it is consistently found that the quark condensate does not vanish in the chiral limit, and that there exists a $\pi$-like particle which becomes very light at small $m$. This result is supported by data for $S U(2)$ and $S U(3)$ gauge theories, and in the quenched as well as the unquenched simulations.

Although all groups agree that the $\pi$-mass becomes very small, it was however observed in [4], and confirmed in [5] through high precision measurements in quenched SU(2) simulations, that a linearextrapolation $m_{\pi}^{2}=s m$ was not adequate in the range $m=0.0125-0.1$, the ratio $m_{\pi}^{2} / m$ exhibiting there a substantial positive curvature. Related observations (non vanishing at $m=0$ of linear fits to the - measured $\pi$-masses) have also been reported for quenched SU(3) [6] and unquenched SU(2) [7]. Among possible explanations for this finding (Ref. [5] excludes the existence of finite size effects at $\left.\beta_{\mathrm{SU}(2)} \equiv 4 / g^{2}=2.3\right)$, it was suggested $[5,8]$ that it 
might be a manifestation of the above described logarithmic singularities. This explanation however contradicts the naive expectation that the absence of internal quark loops in the quenched approximation forbids the occurrence of $\pi$-loops in Green's functions, thus suppressing the long range fluctuations responsible for chiral logarithms. This paper is mainly devoted to disprove this (too) naive statement; it concludes to the existence of such logarithms in the quenched approximation as well as in full QCD.

A strong coupling method for investigating quenched QCD is set up in section 2. Section 3 is devoted to the study of the chiral limit of this model, which is shown to contain chiral logarithms generically. The same conclusion is reached in section 4 from the behaviour at low $m$ of the $\pi$-mass squared measured in numerical simulations of quenched SU(2). Conclusions are proposed in section 5.

\section{A strong coupling calculation. Effective actions.}

In this section, we adapt to the quenched case the strong coupling, large $d$ method ( $d$ =dimension) devised in [9], in order to investigate the behaviour of $m_{\pi}^{2}$ and $f_{\pi}^{2}$ at low $m$. Of course the strong coupling limit is not the right limit to be taken. But spontaneous symmetry breaking, which is known to occur for all values of the lattice coupling, is the basic mechanism for the appearance of infrared logarithms; so we believe that the result of the strong coupling study should be instructive enough, at the qualitative level at least, to shed light also on the continuum limit.

At $\beta=0$, for an $\mathrm{SU}(N)$ gauge symmetry, the lattice partition function is

$$
Z=\int[\mathrm{d} \mu(A)] \exp \left[-S_{\mathrm{KS}}(A)\right],
$$

where $A$ collectively denotes the fermion fields $\chi_{i}(x), \bar{\chi}_{i}(x)\left(i=\right.$ colour index,$x=\left(x_{1}, \ldots, x_{d}\right)$ is the lattice site), and the gauge group elements $U_{\mu}(x)$ sitting on the link $[x, x+a \hat{\mu}]$. The lattice spacing a will be taken equal to unity unless specified. The Kogut-Susskind action is

$$
\begin{aligned}
S_{\mathrm{KS}} \equiv & -\bar{\chi}\left(\not D_{\mathrm{U}}+m\right) \chi=-\frac{1}{2} \sum_{x, \mu}(-)^{x_{1}+\cdots+x_{\mu-1}} \times \\
& \times\left[\bar{\chi}(x) U_{\mu}(x) \chi(x+\hat{\mu})\right. \\
& \left.-\bar{\chi}(x+\mu) U_{\mu}^{\dagger}(x) \chi(x)\right]-m \sum_{x} \bar{\chi}(x) \chi(x) .
\end{aligned}
$$

A source term $\sum_{x} J_{x} \bar{\chi}(x) \chi(x)$ can be added, in such a way that mesonic correlation functions are easily derived by differentiating $\ln Z(m, J)$ with respect to $m$ and $J$. Colour indices are omitted. As it stands, the partition function $Z$ is that of full (unquenched) lattice QCD at $\beta=0$. In particular, the condensate $\langle\bar{\chi}(x) \chi(x)\rangle$, an order parameter for the remnant chiral symmetry generator, is given in this case by

$$
\langle\bar{\chi} \chi\rangle_{U Q}=\frac{\int[\mathrm{d} \mu(U)]\left(\not D_{U}+m\right)_{x x}^{-1} \operatorname{det}\left(\not D_{U}+m\right)}{\int[\mathrm{d} \mu(U)] \operatorname{det}\left(\not D_{U}+m\right)},
$$

whereas its quenched value is

$$
\langle\bar{\chi} \chi\rangle_{Q}=\int[\mathrm{d} \mu(U)]\left(\not \emptyset_{U}+m\right)_{x x}^{-1} \text {. }
$$

A way to suppress the fermionic determinant, in order to go from equation (3) to equation (4), is to add the action $S_{\mathrm{KS}}$ a similar action for commuting complex field variables $\varphi_{i}(x)$ in the same representation of the gauge group. It is convenient to write the action

$$
S=-\bar{\chi}\left(\not D_{U}+m\right) \chi+\varphi^{\dagger}\left(\not D_{U}+\mu\right) \varphi,
$$

so that the large positive $\mu$ limit of $Z$, which contains $\exp \left[-\mu \varphi^{\dagger} \varphi\right]$, makes sense under integration over the $\varphi$ variables. The $\varphi$ integration yields $1 /$ det $\left(\not D_{U}+\mu\right)$, which just cancels the fermionic determinant at $m=\mu$. One has

$$
\langle\bar{\chi} \chi\rangle_{Q}=\left.\frac{\partial}{\partial m} \log Z(m, \mu)\right|_{m=\mu} .
$$

We will now follow the steps described in [9], and compute the quenched partition function. Discussions of possible subsequent corrections in $\beta$ and $1 / d$ can be found in the latter reference.

2.1 INTEGRATION OVER THE GAUGE FIELDS. Integrating first over the gauge fields yields an effective action which depends on the gauge invariants which can be built out of the $\varphi, \varphi^{\dagger}$, $\chi, \bar{\chi}$ variables. Choosing normalizations for later convenience, we then introduce

$$
\begin{gathered}
M_{x}=\frac{1}{N} \sqrt{\frac{d}{2}} \bar{\chi}(x) \chi(x) \\
H_{x}=\frac{1}{N} \sqrt{\frac{d}{2} \varphi^{\dagger}(x) \varphi(x)} \\
\bar{F}_{x}=\frac{1}{N} \sqrt{\frac{d}{2}} \bar{\chi}(x) \varphi(x) ; \\
F_{x}=\frac{1}{N} \sqrt{\frac{d}{2}} \varphi^{\dagger}(x) \chi(x) .
\end{gathered}
$$

Summation over the colour indices is always understood. There are other gauge invariants such as baryons and mixed composite operators (containing $\chi$ 's and $\varphi$ 's), which are antisymmetric in colour 
space. For SU $(N>2)$ however, they give contributions of lower order in $1 / d$, the $S U(2)$ case being special [10]. It will be discussed later on.

The result of the one link integral to be performed is known under the form of an expansion in powers of the composite fields. Again we are interested in the leading contribution in $1 / d$, which corresponds to keeping only the first term of this expansion, quadratic in $M, H, \bar{F}, F$. One obtains

$$
\begin{aligned}
& Z=\int[\mathrm{d} \chi\left.\mathrm{d} \bar{\chi} \mathrm{d} \varphi \mathrm{d} \varphi^{\dagger}\right] \exp \left\{N \left[\frac{M V M}{2}-\right.\right. \\
&\left.\left.-\frac{H V H}{2}+\bar{F} V F+2 \bar{m} M-2 \bar{\mu} H\right]\right\}
\end{aligned}
$$

In this representation, we have set

$$
\bar{m}=m /(2 d)^{1 / 2} ; \quad \bar{\mu}=\mu /(2 d)^{1 / 2},
$$

and the interaction $V$ is the nearest neighbour interaction

$$
V_{x x^{\prime}}=\frac{1}{2 d} \sum_{\mu}\left(\delta_{x, x^{\prime}+\hat{\mu}}+\delta_{x, x^{\prime}-\hat{\mu}}\right),
$$

which reads in momentum space

$$
V_{q}=\frac{1}{d} \sum_{\mu} \cos q_{\mu} .
$$

The momentum $q_{\mu}$ lies in the first Brillouin zone $-\pi<q_{\mu} \leqslant \pi$, and the potential $V$ is bound between $-1\left(q_{\mu}=\pi\right)$ and $+1\left(q_{\mu}=0\right)$. As will be seen later, the occurrence of Goldstone modes will follow from a lowest order boson propagator of the form $\left(V_{q}+1\right)^{-1}$ at $m=0$, which has a pole at $q_{\mu}=\pi$.

\subsection{LAPLACE TRANSFORMS AND INTEGRATION} OVER THE ELEMENTARY MATTER FIELDS. - The next step consists in making a Laplace transform of the exponential appearing in equation (8), in such a way that the resulting representation eventually is quadratic in the $\chi$ and $\varphi$ fields, and suitable for integration over these variables. Up to constant factors, the resulting functional integral is

$$
\begin{array}{r}
Z=\int[\mathrm{d} \lambda \mathrm{d} \sigma \mathrm{d} \Lambda \mathrm{d} \bar{\Lambda}] z \exp \left\{-\frac{N}{2} \lambda V^{-1} \lambda+\right. \\
\left.+\frac{N}{2} \sigma V^{-1} \sigma-N \bar{\Lambda} V^{-1} \Lambda\right\},
\end{array}
$$

where $z$ contains the integration over the $\chi, \varphi$ fields :

$$
\begin{aligned}
z= & \prod_{x} \int\left[\mathrm{d} \chi \mathrm{d} \bar{\chi} \mathrm{d} \varphi \mathrm{d} \varphi^{\dagger} l_{x} \times\right. \\
& \times \exp \left\{N\left(\lambda_{x}+2 \bar{m}\right) M_{x}-\right. \\
-N\left(\sigma_{x}+2 \bar{\mu}\right) H_{x}- & \left.N \bar{\Lambda}_{x} F_{x}-N \bar{F}_{x} \Lambda_{x}\right\} .
\end{aligned}
$$

In equation (12), the quadratic form in $\lambda, \sigma, \bar{\Lambda}, \Lambda$ is proportional to the inverse of the quadratic form appearing in (8) for the conjugate variables, respectively $M, H, F, \bar{F}$. The functional integral (13) is easy to perform because all the elementary fields appear in single site, quadratic composite operators. Owing to the Grassmann character of $\bar{\Lambda}$ and $\Lambda$, one may further reduce $\ln z$ to its linear part in $\bar{\Lambda}_{x} \Lambda_{x}$, and immediately integrate (12) over these variables. Restoring a source term $N \sum_{x} j_{x} M_{x}$ in the initial action, and after a translation $\lambda_{x} \rightarrow \lambda_{x}-$ $2 \bar{m}-j(x), \sigma_{x} \rightarrow \sigma_{x}-2 \bar{\mu}$, the final result for $Z$ (quenched) can be cast into the form of the following functional integral over the real scalar fields $\lambda$ and $\sigma:$

$$
\begin{aligned}
& Z_{Q}\left(j_{x}, m, \mu\right)=\int[\mathrm{d} \lambda \mathrm{d} \sigma] \exp \left\{-S_{\mathrm{eff}}(\lambda, \sigma)\right\} \\
& S_{\text {eff }}(\lambda, \sigma)= \frac{N}{2}\left[(\lambda-2 \bar{m}-j) V^{-1}(\lambda-2 \bar{m}-j)\right. \\
&\left.-(\sigma-2 \bar{\mu}) V^{-1}(\sigma-2 \bar{\mu})\right] \\
&-N \sum_{x}\left(\ln \lambda_{x}-\ln \sigma_{x}\right) \\
&-\operatorname{Tr} \ln \left[V^{-1}+\frac{1}{\lambda \sigma}\right] .
\end{aligned}
$$

In the last term, which comes from the integration over $\bar{\Lambda}, \Lambda$, the operator appearing under $\operatorname{Tr} \ln$ has to be understood as

$$
\left(V^{-1}+\frac{1}{\lambda \sigma}\right)_{x y}=V_{x y}^{-1}+\frac{1}{\lambda_{x} \sigma_{x}} \delta_{x y} .
$$

The unquenched partition function is of course simpler (no $\varphi$ field, and thus no $\sigma$ field) :

$$
\begin{aligned}
& Z_{U Q}(j, m)=\int \mathrm{d} \lambda \times \\
& \quad \times \exp \left\{-\frac{N}{2}(\lambda-2 \bar{m}-j) V^{-1}(\lambda-2 \bar{m}-j)+\right. \\
& \left.+N \sum_{x} \ln \lambda_{x}\right\} \cdot(16)
\end{aligned}
$$

The forms reached for $Z_{Q}$ and $Z_{U Q}$ are suitable for a study by saddle point methods. In particular, we have to deal with fields $\sigma$ and $\lambda$ with flat measures.

\subsection{SADDLE POINT TREATMENT OF THE PARTITION} FUNCTIONS. - We will start with the action $S_{\text {eff }}^{0}(\lambda, \sigma)$ obtained from the quenched action of equation (14) without the $\operatorname{Tr} \ln$ coming from $\bar{\Lambda}, \Lambda$ integration (no loop at all). It is easy to find that this action $S^{0}$ is stationary for

$$
\begin{aligned}
& \lambda_{x}=\bar{\lambda}=\bar{m}+\left(\bar{m}^{2}+1\right)^{1 / 2} \\
& \sigma_{x}=\bar{\sigma}=\bar{\mu}+\left(\bar{\mu}^{2}+1\right)^{1 / 2} .
\end{aligned}
$$

Choosing the + sign in front of the square root in 
$\bar{\lambda}$ is straightforward : it just correspond to minimizing the action with respect to $\lambda_{x}$ for $m>0$. One has to be careful in choosing the same sign for $\bar{\sigma}$. This originates from the positivity of the $H$ operator (Eq. (7)) appearing in equation (13): the $\varphi, \varphi^{\dagger}$ integration makes sense for $\sigma_{x}+2 \bar{\mu}>0$. A less formal treatment of the Laplace transform (in fact starting from a Fourier transform and looking at the proper contours to be chosen) confirms this result. Note that $\langle H\rangle$ is given by $-n_{\mathrm{s}}^{-1} \frac{1}{N} \frac{\partial}{\partial \bar{\mu}} \ln Z$, equal to $+(\bar{\sigma}-2 \bar{\mu})$ from (14) and indeed positive for the choice (17) ( $n_{\mathrm{s}}$ is the number of lattice sites).

Next we integrate the quadratic fluctuations in $\lambda_{x}$ and $\sigma_{x}$ around the saddle point (17), add to the resulting action the previously omitted $\bar{\Lambda}, \Lambda$ loop contribution, to obtain the following complete one loop expression for $\ln Z$ at the saddle point

$$
\begin{aligned}
& \frac{1}{n_{\mathrm{s}}} W_{\mathrm{Q}}^{1}(\bar{m}, \bar{\mu}) \equiv \frac{\ln Z}{n_{\mathrm{s}}}= \\
& \quad-\frac{N}{2}\left[(\bar{\lambda}-2 \bar{m})^{2}-(\bar{\sigma}-2 \bar{\mu})^{2}\right] \\
& +N \ln \bar{\lambda}-N \ln \bar{\sigma}-\frac{1}{2 n_{\mathrm{s}}} \operatorname{Tr} \ln \left(V^{-1}+\frac{1}{\lambda^{2}}\right) \\
& \quad-\frac{1}{2 n_{\mathrm{s}}} \operatorname{Tr} \ln \left(V^{-1}+\frac{1}{\bar{\sigma}^{2}}\right) \\
& \quad+\frac{1}{n_{\mathrm{s}}} \operatorname{Tr} \ln \left(V^{-1}+\frac{1}{\bar{\lambda} \bar{\sigma}}\right) .
\end{aligned}
$$

The various contributions appearing in this expression are easy to interpret. The first two terms directly reflect the quadratic parts in $M$ and $H$ appearing in equation (8). (Note that $\sum_{x} V_{x x^{\prime}}=1$.)

The next two ones represent the result of integrating the respectively anti-commuting and commuting $(\chi, \bar{\chi})$ and $\left(\varphi, \varphi^{\dagger}\right)$ variables (see (13)). Finally, the $\operatorname{Tr} \ln$ terms are the one loop contributions of 2 real scalar fields $\left(\lambda_{x}-\bar{\lambda}, \sigma_{x}-\bar{\sigma}\right)$ and of one complex Grassmann field $(\bar{\Lambda}, \Lambda)$, with the respective lowest order inverse propagators

$$
V^{-1}+\frac{1}{\bar{\lambda}^{2}}, V^{-1}+\frac{1}{\bar{\sigma}^{2}}, V^{-1}+\frac{1}{\bar{\lambda} \bar{\sigma}}
$$

We are now going to exploit the basic result (18), where the main feature of interest for our purpose is that the above inverse propagators all vanish in the chiral limit $m=\mu=0$ for $q_{\mu}=\pi$ (3 zero modes). Of course, in the unquenched case, equation (18) is replaced by

$$
\begin{aligned}
& \frac{1}{n_{\mathrm{s}}} W_{U Q}^{1}(\bar{m}, \bar{\mu})=-\frac{N}{2}(\bar{\lambda}-2 \bar{m})^{2}+ \\
& \quad+N \ln \bar{\lambda}-\frac{1}{2 n_{\mathrm{s}}} \operatorname{Tr} \ln \left(V^{-1}+\frac{1}{\bar{\lambda}^{2}}\right),
\end{aligned}
$$

\section{Analysis of the strong coupling result.}

The results $(18,19)$, valid in the absence of local sources $j_{x}$, allows us to compute the vacuum expectation value $\langle\bar{\chi} \chi\rangle$ at one loop order. We will next investigate a two point meson correlation function in order to study the $\pi$ mass.

3.1 The QuARK CONDENSATE. - From equation (2), we have

$$
\langle\bar{\chi} \chi\rangle=\frac{1}{n_{\mathrm{s}}} \frac{\partial}{\partial m} \ln Z .
$$

Let us start with the (simpler) unquenched case. Equation (19) immediately tells us (recall that $\left.\bar{m}=\frac{m}{\sqrt{2 d}}\right)$

$$
\langle\bar{\chi} \chi\rangle_{U Q}=\frac{2 N}{\sqrt{2 d}}(\bar{\lambda}-2 \bar{m})+\frac{1}{\bar{\lambda} \sqrt{2 d}} \frac{\partial \bar{\lambda}}{\partial \bar{m}} d_{0},
$$

with

$$
d_{0}=\int \frac{\mathrm{d}^{d} q}{(2 \pi)^{d}} D_{0}(q),
$$

where we defined the zeroth order propagator $D_{0}$ to be

$$
D_{0}=\frac{1}{\bar{\lambda}^{2}}\left(V_{q}^{-1}+\frac{1}{\bar{\lambda}^{2}}\right)^{-1}=V_{q}\left(V_{q}+\bar{\lambda}^{2}\right)^{-1} \text {. }
$$

For $m$ fixed $\neq 0, \bar{\lambda}^{2}$ is larger than $1,\left|V_{q}\right|$ is bounded by 1 (Eq. (11)), and $D_{0}(q)$ has no pole in the integration domain. The quantity $d_{0}$ can be expanded in powers of $1 / d$, and appears as a $1 / d$ correction to the lowest order term [9]

$$
\langle\bar{\chi} \chi\rangle_{U Q}^{(0)}=N \sqrt{\frac{2}{d}}(\bar{\lambda}-2 \bar{m}) .
$$

There are other corrections of order $1 / d$, coming from terms of the link integral which we have neglected in equation (8), but they are regular in the chiral limit. Let us now evaluate the singular part of $d_{0}$ as $m \rightarrow 0$, a case where $\bar{\lambda} \rightarrow 1$, so that the inverse propagator tends to vanish at $q_{\mu}=\pi$. We set $q_{\mu}=\pi+k_{\mu}, M_{0}^{2}=2 d\left(\bar{\lambda}^{2}-1\right)$ (the squared pion mass) which behaves as

$$
M_{0}^{2} \sim s m=2 \sqrt{2 d} m
$$

in the chiral limit. Then the quantity $d_{0}$ reads

$$
d_{0}=-2 d \int \frac{\mathrm{d}^{d} k}{(2 \pi)^{-d}} \frac{V_{k}}{M_{0}^{2}+2 d\left(1-V_{k}\right)},
$$

and the integrand becomes singular at $k=0$, $M_{0}^{2} \rightarrow 0$. Hence the singular part of $d_{0}$ is that of

$$
d_{0}^{\mathrm{s}}=-2 d \int_{(\varepsilon)} \frac{\mathrm{d}^{d} k}{(2 \pi)^{d}} \frac{1}{M_{0}^{2}+k^{2}},
$$


where the symbol $(\varepsilon)$ means one integrates inside a small $d$-dimensional sphere of radius $\varepsilon$. One finds that the leading singular part is (for $d=4$ )

$$
d_{0}^{\mathrm{s}}=-\frac{1}{2 \pi^{2}} M_{0}^{2} \ln M_{0}^{2} \text {. }
$$

(In $\operatorname{dim} . d$, the derivative of order $(d-2) / 2$ with respect to $M_{0}^{2}$ is logarithmically divergent). Then equation (21) can be rewritten

$$
\begin{aligned}
& \langle\bar{\chi} \chi\rangle_{U Q}^{(1)}= \\
& \quad=\langle\bar{\chi} \chi\rangle^{(0)}\left[1-\frac{1}{4 N \pi^{2}} M_{0}^{2} \ln M_{0}^{2}+\text { s.t. }\right]
\end{aligned}
$$

Here s.t. (smaller terms) denotes contributions which are less singular and/or smaller by powers of $1 / d$. We further remark that, after defining a $\pi$ decay constant $F_{0}$ at lowest order by

$$
F_{0}^{2}=\frac{m\langle\bar{\chi} \chi\rangle^{(0)}}{M_{0}^{2}}
$$

which leads (Eqs. (21) and (24)) to

$$
F_{0}^{2}=N / 2 d,
$$

equation (28) can be consistently written as

$$
\begin{aligned}
\langle\bar{\chi} \chi\rangle_{U Q}^{(1)} & = \\
& =\langle\bar{\chi} \chi\rangle^{0}\left[1-\frac{1}{32 \pi^{2}} \frac{M_{0}^{2}}{F_{0}^{2}} \ln M_{0}^{2}+\text { s.t. }\right] .
\end{aligned}
$$

This result is in agreement with the general result that the logarithmic correction is proportional to $M_{0}^{2} / F_{0}^{2}$ [3]. Let us now investigate the quenched case along the same lines.

We start from equation (18) and differentiate with respect to $m$ to find

$$
\begin{aligned}
&\langle\bar{x} \chi\rangle_{Q}=\frac{2 N}{\sqrt{2 d}}(\bar{\lambda}-2 \bar{m})+ \\
&+\frac{1}{\sqrt{2 d}} \frac{\partial \bar{\lambda}}{\partial \bar{m}}\left[\frac{1}{\bar{\lambda}} d_{0}-\frac{1}{\bar{\lambda}^{2} \bar{\sigma}} \frac{\operatorname{Tr}}{n_{\mathrm{s}}}\left(V^{-1}+\frac{1}{\bar{\lambda} \bar{\sigma}}\right)^{-1}\right] .
\end{aligned}
$$

Taking finally $m=\mu$ in this result, we have $\bar{\lambda}=\bar{\sigma}$ and, due to $(22,23)$, we discover that the two corrections coming from $\lambda$ and $\Lambda$ loops exactly cancel each other (at this order in $\beta$ and $1 / d$ and for $\mathrm{SU}(N>2))$. There is no contribution from the $\sigma$ loop since it is independent of $\bar{m}$.

Hence, in this case, the naive expectation that the absence of internal quark loop induces the absence of $\pi$-loops, and thus of chiral logarithms, seems to be correct. More precisely in fact, the $\lambda$-loop contribution (which reflects the $\pi$-propagation since $\pi$ couples to $\bar{\chi} \chi$, conjugate to $\lambda$ ) is cancelled by the
$\Lambda, \bar{\Lambda}$ loop contribution, to be attributed to the effect of the $\bar{\chi} \varphi$ and $\varphi^{\dagger} \chi$ operators introduced by quenching. It is not guaranteed that the same cancellation occurs in other Green's functions, and we will actually see now that it does not in general, by computing the $\pi$-mass at 1 -loop order.

3.2. THE $\pi$-PROPAGATOR AT ONE LOOP ORDER. The $\pi$-mass can be computed by looking at the poles of the $M_{x}$ fields (Eq. (7)) connected 2-point function in momentum space. From (14) or (16) we obtain

$$
\Delta_{M} \equiv N\langle M M\rangle_{\mathrm{C}}=-V^{-1}+V^{-1} \Delta_{\lambda} V^{-1},
$$

with $\Delta_{\lambda}=N\langle\lambda \lambda\rangle_{c}$. At lowest order (no loop), $\Delta_{\lambda}$ is just

$$
\Delta_{\lambda}^{0}=\bar{\lambda}^{2} D_{0}=\bar{\lambda}^{2} V\left(V+\bar{\lambda}^{2}\right)^{-1},
$$

which gives

$$
\Delta_{M}^{0}(q)=-\left(\bar{\lambda}^{2}+V_{q}\right)^{-1} .
$$

We already saw in section (3.1) that this propagator has a pole corresponding in the chiral limit to a mass $M_{0}$,

$$
M_{0}^{2}=2 d\left(\bar{\lambda}^{2}-1\right) \simeq 2 \sqrt{2 d} m+O\left(m^{2}\right) .
$$

We are interested in the corrections to $M_{0}$ coming from the large fluctuations induced by the $m \rightarrow 0$ limit, and associated with the one loop contributions in the $\lambda, \sigma$ and $\bar{\Lambda}, \Lambda$ fields. To this end, equation (31) tells us that we have to find the zeroes of the inverse $\lambda$ propagator at one loop order, for each of the two, quenched and unquenched, cases. With the notations of equations (22), (23), we have obtained the following results

$$
\begin{array}{r}
\langle\lambda \lambda\rangle_{C, Q}^{-1}=\frac{N}{\bar{\lambda}^{2}}\left[D_{0}^{-1}-\frac{1}{N}\left(D_{0} * D_{0}-d_{0}\right)\right] \\
\langle\lambda \lambda\rangle_{C, \cup Q}^{-1}=\frac{N}{\bar{\lambda}^{2}}\left[D_{0}^{-1}-\frac{1}{N}\left(2\left(D_{0} * D_{0}-d_{0}\right)-\right.\right. \\
\left.-\frac{d_{0}}{\bar{\lambda}^{2}+1}\left(\bar{\lambda}^{2}-1\right)\right]
\end{array}
$$

The expression $D_{0} * D_{0}$ means convolution in momentum space, that is $\left(D_{0} * D_{0}\right)_{x y}=\left(D_{0_{x y}}\right)^{2}$, or $(d=4)$

$$
\begin{array}{r}
\left(D_{0} * D_{0}\right)_{p}=\int \frac{\mathrm{d}^{4} k}{(2 \pi)^{4}} \frac{V_{k}}{\bar{\lambda}^{2}}\left(1+\frac{V_{k}}{\bar{\lambda}^{2}}\right)^{-1} \times \\
\times \frac{V_{p+k}}{\bar{\lambda}^{2}}\left(1+\frac{V_{p+k}}{\bar{\lambda}^{2}}\right)^{-1},
\end{array}
$$

with $V_{h}=\frac{1}{4} \sum_{\mu} \cosh _{\mu}$.

Before actual computations of singular contributions, let us make the following comments on 
the low momentum, low $m$ behaviour of the $\lambda$ propagator.

(i) Since we are going to find the pion as a pole close to $p_{\mu}=\pi$ in the chiral limit, we set $p_{\mu}=\pi+q_{\mu}$. We next show that the quantity $X_{q}=D_{0} * D_{0}-d_{0}$ appearing in equations (35) and (36) vanishes at $q_{\mu}=0$. Some manipulations indeed lead to

$X_{q}=-\int \frac{\mathrm{d}^{4} k}{(2 \pi)^{4}} \frac{V_{k}}{\bar{\lambda}^{2}}\left(1+\frac{V_{k}}{\bar{\lambda}^{2}}\right)^{-1}\left(1-\frac{V_{k+q}}{\bar{\lambda}^{2}}\right)^{-1}$.

The integration domain is $-\pi<k_{\mu} \leqslant \pi$ (or any domain of length $2 \pi$ ). At $q=0$, the integrand is odd under $k_{\mu} \rightarrow k_{\mu}+\pi$, and thus $X_{q=0}=0$. Since furthermore $X_{q}=X_{-q}$ (the integrand is invariant under $k \rightarrow-k, q \rightarrow-q$ ), we deduce that $X_{q}$ is of order $q^{2}$ at low $q$.

(ii) In equation (35), the second part of the correction to $D_{0}^{-1}$, the zeroth order inverse propagator, is proportional to $\bar{\lambda}^{2}-1$, and thus vanishes linearly in $m$, or $M_{0}^{2}$. Hence the corrections are proportional to either $q^{2}$ or $M_{0}^{2}$, which insures that the pole of $\langle\lambda \lambda\rangle$ is still located at 0 as $m \rightarrow 0$. This has to be so of course since $\langle\bar{\chi} \chi\rangle$ is still different from zero (section 3.1).

(iii) There is a Ward identify associated with the chiral symmetry of the model [11]. In the $\lambda$ variables, it reads

$$
\begin{aligned}
\langle\lambda\rangle & =-2 N \bar{m} \sum_{x}\left\langle\lambda_{x} \lambda_{0}\right\rangle(-)^{x} \\
& =-2 N \bar{m}\langle\lambda \lambda\rangle_{c}\left(p_{\mu}=\pi\right) .
\end{aligned}
$$

A (partial) check of our calculation consists in verifying that this identity is fulfilled by our results. It is particularly easy in the quenched case where $\langle\lambda\rangle=\bar{\lambda}$ even at one loop. We must have from (34), (37) and the vanishing of $X_{q=0}$ (point (ii) above)

$$
\bar{\lambda}=-2 N \bar{m} \times \frac{\bar{\lambda}^{2}}{N} D_{0}\left(p_{\mu}=\pi\right) .
$$

That this is true follows from the expression (23) of $D_{0}$ and the value (17) of $\bar{\lambda}$ :

$$
D_{0}(p=\pi)=-1 \frac{1}{\bar{\lambda}^{2}-1}=\frac{-1}{2 \bar{m} \bar{\lambda}}
$$

The absence of a term proportional to $\bar{\lambda}^{2}$ in the quenched propagator (34) makes consistent with each other the presence of a logarithm in $m_{\pi}^{2}$ and its absence in $\langle\bar{\chi} \chi\rangle_{Q}$.

3.3 CHIRAL LOGARITHMS IN $m_{\pi}^{2}$. - We obtain $m_{\pi}^{2}$ at one loop by looking for the location of the zero of either (34) or (35) which lies close to $p=\pi$. Consider the quenched case first. The correc- tion to $D_{0}^{-1}(p)$ in the inverse propagator is, for $p_{\mu}=\pi+q_{\mu}$

$$
\begin{gathered}
-\frac{1}{N}\left(D_{0} * D_{0}-d_{0}\right)_{p}=\frac{1}{N \bar{\lambda}^{2}} I(q) \\
I(q)=\int \frac{\mathrm{d}^{4} k}{(2 \pi)^{4}} \frac{V_{k}}{\left(1+\frac{V_{k}}{\bar{\lambda}^{2}}\right)} \frac{1}{\left(1-\frac{V_{k+q}}{\bar{\lambda}^{2}}\right)} .
\end{gathered}
$$

Again for any fixed $m \neq 0$, one has $\bar{\lambda}^{2}>1$, and the integral is perfectly well behaved. A logarithmic singularity occurs as $m \rightarrow 0, \bar{\lambda} \rightarrow 1$, which comes from either of the two propagators in (38), when $k_{\mu}$ approaches $\pi$ or $-q_{\mu}$. The singular part of $I(q)$ thus is the same as that of

$$
\begin{aligned}
I^{\mathrm{s}}(q) & =\frac{V_{q}-1}{1+V_{q} / \bar{\lambda}^{2}} \int_{(\varepsilon)} \frac{\mathrm{d}^{4} h}{(2 \pi)^{4}}\left(1-V_{h} / \bar{\lambda}^{2}\right)^{-1} \\
& =\frac{8 \bar{\lambda}^{2}\left(V_{q}-1\right)}{1+V_{q} / \lambda^{2}} \int_{(\varepsilon)} \frac{\mathrm{d}^{4} h}{(2 \pi)^{4}} \frac{1}{h^{2}+M_{0}^{2}}
\end{aligned}
$$

where again $(\varepsilon)$ denotes a small sphere of radius $\varepsilon$, and $M_{0}^{2}$ is the lowest order $\pi$ mass squared $8\left(\bar{\lambda}^{2}-1\right)$. For $\bar{\lambda}$ close to 1 , one finds

$I^{\mathrm{s}}(q)=\frac{\bar{\lambda}^{2}\left(V_{q}-1\right)}{1+V_{q} / \bar{\lambda}^{2}} \frac{1}{2 \pi^{2}} M_{0}^{2} \ln M_{0}^{2}+$ smaller terms.

Plugging in this expression in (34) leads to the following logarithmic singularity in the $\pi$-mass squared

$$
m_{\pi}^{2}(m)_{Q}=M_{0}^{2}\left[1-\frac{1}{N} \frac{1}{(2 \pi)^{2}} M_{0}^{2} \ln M_{0}^{2}\right]
$$

an expression which is valid up to regular, or smaller singular terms in $M_{0}^{2}$. We also recall that it has been obtained by setting $d=4$ in the result of a $1 / d$ expansion ; the numerical coefficients in the slope of $M_{0}^{2}$ versus $m$, and in front of the logarithm, must be understood in this sense. The same remark applies to the following result obtained for the unquenched case (Eq. (35))

$$
m_{\pi}^{2}(m)_{U Q}=M_{0}^{2}\left[1-\frac{1}{N} \frac{1}{(2 \pi)^{2}} M_{0}^{2} \ln M_{0}^{2}\right],
$$

that is... the same result as in the quenched case.

We do not know whether this equality of the $Q$ and $U Q$ corrections is a general result for $m_{\pi}^{2}$, or an accident of the peculiar approximation pattern ( $\beta=0,1 / d$ expansion). It is however clear that it is not a general property for all quantities since we found different logarithmic corrections for $\langle\bar{x} \chi\rangle$, a difference which is apparent in the distinct forms of the one loop $\lambda$ propagator (34) and (35). 
3.4 The CASE of SU(2) GaUge theory. - The peculiarities of the $\mathrm{SU}(2)$ gauge theory are due to the fact that the 2 and $\overline{2}$ representations are equivalent. So the chiral symmetry group is larger, and its spontaneous breakdown leads to more Goldstone particles in the chiral limit [10]. In the unquenched case, there are 3 Goldstone modes which are excited by the composite operator $\bar{\chi}_{i} \chi_{i}$ (as before) and by the 2 baryonic operators $\varepsilon_{i j} \chi_{i} \chi_{j}$ and $\varepsilon_{i j} \bar{\chi}_{i} \bar{\chi}_{j}$. In performing the quenched analysis, that is introducing the $\varphi, \varphi^{\dagger}$ fields in the $2, \overline{2}$ SU (2) representations, one still enlarges the number of zero modes. The operators containing $\varphi$ or $\varphi^{\dagger}$ and involved in the problem of chiral logarithms are $\varepsilon_{i j} \bar{\chi}_{i} \varphi_{j}^{*}, \varepsilon_{i j} \chi_{i} \varphi_{j}$ in addition to $\bar{\chi}_{i} \varphi_{i}$ and $\varphi_{i}^{\dagger} \chi_{i}$. We next proceed along the same steps as for $N>2$ : introduction of variables conjugate to each of the relevant operators, integration over the $\chi$ 's and $\varphi$ 's, saddle point treatment of the effective theory obtained.

The unquenched result of these calculations is easy to understand. Instead of 1 Goldstone boson associated with $\bar{\chi} \chi$, there are 3 of them, and with respect to the SU(N) result with $N$ set equal to 2 , the loop contribution is multiplied by 3 : we obtain, instead of (21) and (42),

$$
\mathrm{SU}(2):\left\{\begin{aligned}
\langle\bar{\chi} \chi\rangle_{U Q}= & \frac{2 N}{\sqrt{2 d}}(\bar{\lambda}-2 \bar{m})+ \\
& +\frac{1}{\bar{\lambda}} \frac{\partial \bar{\lambda}}{\sqrt{2 d} \partial \bar{m}} \times 3 d_{0} \\
m_{\pi}^{2}(m)_{U Q} & =M_{0}^{2}\left[1-\frac{3}{N} \frac{1}{(2 \pi)^{2}} M_{0}^{2} \ln M_{0}^{2}\right] .
\end{aligned}\right.
$$

The SU(2), quenched result, is obtained by actual calculation. We find :

$$
\mathrm{SU}(2):\left\{\begin{aligned}
\langle\bar{\chi} \chi\rangle_{Q}= & \frac{2 N}{\sqrt{2 d}}(\bar{\lambda}-2 \bar{m})+ \\
& +\frac{1}{\bar{\lambda}} \frac{\partial \bar{\lambda}}{\sqrt{2 d} \partial \bar{m}} \times d_{0} \\
m_{\pi Q}^{2}(m)= & m_{\pi U Q}^{2}(m) .
\end{aligned}\right.
$$

We have no simple explanation for this result namely the occurrence of a partial cancellation only of the log's in $\bar{\chi} \chi$, and again the equality of the logarithmic quenched and unquenched corrections to $M_{0}^{2}$.

Here also, these equations are to be understood in the sense of leading singular contributions to the quantities which are computed. Let us finally mention that all the above results for $\langle\bar{\chi} \chi\rangle$ and $m_{\pi}^{2}$ can be translated into properties for $f_{\pi}^{2}$ and $m_{\pi}^{2}$ using the PCAC relation, valid in the situation just recalled, namely

$$
f_{\pi}^{2} m_{\pi}^{2}=m\langle\bar{\chi} \chi\rangle .
$$

We conclude this section by summarizing as follows what we have learned by the strong coupling investigation : the occurrence of chiral logarithms is a generic feature which shows up in most physical quantities, even in the quenched case. The only exception we have encountered concerns $\langle\bar{\chi} \chi\rangle_{Q}$ for $\mathrm{SU}(N>2)$ at $\beta=0$ and leading order in $1 / d$.

\section{Analysis of numerical results at large $\beta$ values.}

Guided by the above results, we have tempted to interpret in terms of chiral logarithms the non linearities observed for $m_{\pi}^{2}(m)$ in the quenched SU(2) simulation of reference [5]. In particular, it was shown there that at $\beta=2.3$, no finite size effect could be made responsible for our observation. We propose the following parametrization of the measured $m_{\pi}^{2}$ values

$\frac{1}{2}\left[\cosh m_{\pi}-1\right]=s m\left[1+b \frac{m}{m_{0}} \ln \frac{m}{m+m_{0}}\right]$.

Such a formula accounts for the expected form of the logarithmic singularity below $m=m_{0}$, namely $m_{\pi}^{2}=s m\left[1+b \frac{m}{m_{0}} \ln m+0(m)\right]$.

For $m \gg m_{0}$, it is equivalent to

$$
\frac{1}{2}\left[\cosh m_{\pi}-1\right]=s m(1-b)+\frac{s b m_{0}}{2}+0\left(\frac{m_{0}}{m}\right),
$$

that is consistent with the first observation of reference [4] that a linear fit of [ $\left.\cosh m_{\pi}-1\right]$ with a nonvanishing intercept was preferred by the data at $m \geqslant 0.05$. The result [12] of a fit to equation (48) gives (in units of the lattice spacing)

$$
\beta=2.3\left\{\begin{array}{l}
s=9.7 \text { (4) } \\
b=0.33 \text { (3) } \\
m_{0}=0.035(7)
\end{array}\right.
$$

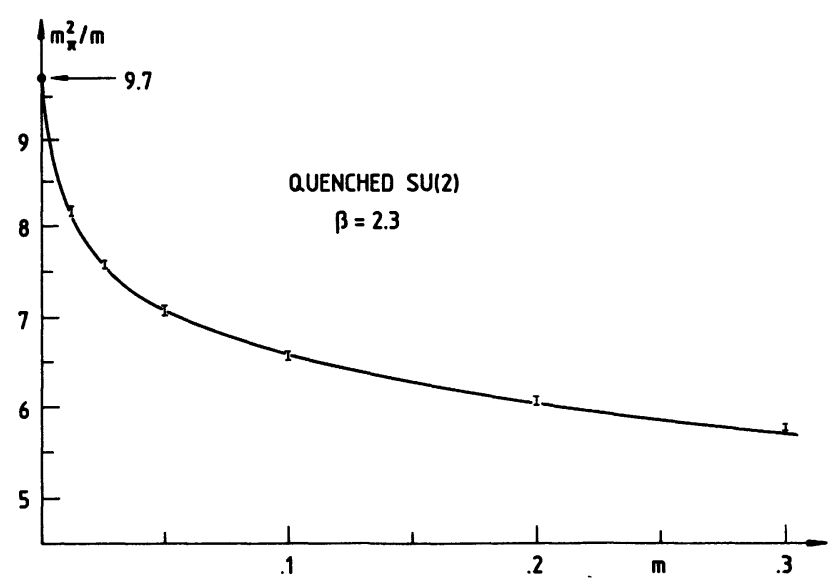

Fig. 1. - Fit of the $m_{\pi}^{2} / m$ data of reference [5] for $\beta=2.3$ to the parametrization of equation (48) with the values of equation (49). 
and leads to an excellent representation of the data (Fig. 1). Although we have some suspicion that the lowest $m$ data at $\beta=2.4$ suffer from finite size effects [5], the same form (48) happens to be also adequate in this case for [12]

$$
\beta=2.4 \begin{cases}s & =8.1 \\ b & =0.24 \\ m_{0} & =0.015\end{cases}
$$

with the result shown in figure 2 .

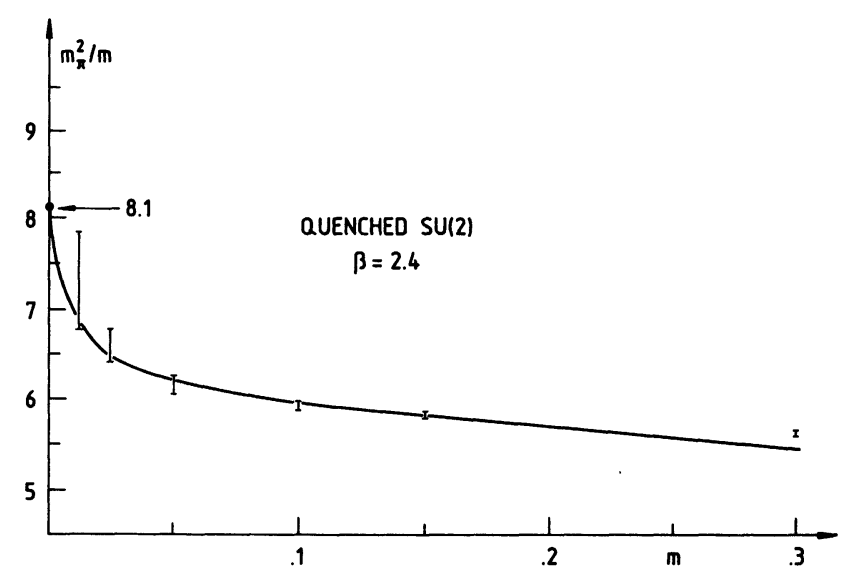

Fig. 2. - Fit of $m_{\pi}^{2} / m$ data of reference [5] for $\beta=2.4$ to the parametrization of equation (48) with the values of equation (50).

As we recalled it in the introduction, the coefficient of the logarithm in $m_{\pi}^{2}$ can be computed in the continuum, with the following result for (non SU(2)) QCD theory. In the case of 2 flavours, the result is [3]

$$
\left\{\begin{array}{l}
m_{\pi}^{2}=M_{0}^{2}\left[1+\alpha \frac{M_{0}^{2}}{F_{0}^{2}} \ln M_{0}^{2}+\cdots\right] \\
\alpha=\frac{1}{32 \pi^{2}}
\end{array}\right.
$$

where, in the non linear $\sigma$-model, $M_{0}^{2}$ is the input $\pi$ mass squared, and $F_{0}^{2}$ the input $\pi$ decay constant squared at $M_{0}^{2}=0$. Within the ansatz (48) we should thus compare $b F_{0}^{2} / s m_{0}$ with $\alpha=1 / 32 \pi^{2}$. We define

$$
\alpha_{\mathrm{SU}(2)}^{\mathrm{Q}}=\frac{b F_{0}^{2}}{s m_{0}},
$$

and obtain from (49), (50) and the values of $f_{\pi}^{2}=F_{0}^{2} / 2$ found in reference [5]

$$
\begin{aligned}
& 32 \pi^{2} \alpha_{\mathrm{SU}(2)}^{\mathrm{Q}}=3.4 \text { at } \beta=2.3 \\
& 32 \pi^{2} \alpha_{\mathrm{SU}(2)}^{\mathrm{Q}}=3.1 \text { at } \beta=2.4 \text {. }
\end{aligned}
$$

A correct evaluation of the error on these numbers would have required a reanalysis of the data in order to compute the fluctuations from bin to bin of the combination (52) of $b, F_{0}^{2}, s$ and $m_{0}$, which were unfortunately measured independently. An error of about $20 \%$ seems to be a reasonable guess. One might be tempted to speculate that

$$
\alpha_{\mathrm{SU}(2)}^{\mathrm{Q}}=3 \alpha
$$

although we have no clear explanation for that. In section 3, we understood such a factor 3 when comparing the unquenched $\mathrm{SU}(2)$ result to the unquenched $\mathrm{SU}(N>2)$ result. Here, on the one hand the data are for quenched $\mathrm{SU}(2)$, on the other hand the flavour content (4 flavours in the continuum limit of the lattice model) is unclear at finite spacing: although there is good evidence that flavour symmetry is being restored at $\beta \geq 2.3$ [13], there is (strictly speaking) only one Goldstone mode contributing to the log's.

\section{Concluding remarks.}

We have shown that the existence of infrared logarithms, associated with the appearance of Goldstone bosons when chiral symmetry is spontaneously broken, survives quenching. This statement is based on the investigation of the strong coupling limit of QCD, in both the unquenched and quenched case. The latter is treated by introducing auxiliary commuting variables $\varphi$ and $\varphi *$ in the same gauge group representations as the Grassmann fields, $\chi, \bar{\chi}$ representing the quarks, and interacting through the same lattice action. By this trick, the fermionic determinant which multiplies the pure gauge partition function is cancelled, so suppressing in Green's functions the internal quark loops of the full QCD theory. But this suppression is found not to produce a similar suppression of the large distance fluctuations responsible for infrared logarithms in the chiral limit. Moreover, we have shown that, at least in the case of quenched SU(2) QCD, these logarithms give substantial contributions to the quark mass dependence of the $\pi$-mass squared. This conclusion solves the problem raised by former observations in numerical simulations of non linearities of $m_{\pi}^{2}(m)$ at very low $m$. A practical consequence of this finding is that much care should be taken in extrapolating numerical data down to the physical mass region; in particular, we expect these effects to strongly affect the determination, from the physical $\pi$ and $\kappa$ masses, of the $u, d, s$ quark masses. Other quantities of interest in this context are $f_{\pi}^{2}, f_{\kappa}^{2}$, or the ratio $\langle\bar{s} s\rangle /\langle\bar{u} u\rangle$ of the strange to non strange quark condensates.

At the theoretical level, the question is : should we have anticipated the above results from more general considerations [14] ? It is true that infrared singularities are quite generally expected whenever a continuous symmetry is spontaneously broken. In the full QCD case, this is beautifully examplified by 
the treatment given by Gasser and Leutwyler [3] of the low momentum, low $m$ Green's functions. A basic ingredient there, however, is the unitarity of the effective low energy model, a property which is not shared, at least in a straightforward way, by quenched QCD. It might be [14] that quenched QCD has to be viewed as a restriction to a smaller sector of a (unitary ?) effective theory involving more states than full QCD. In the framework of the method used in sections 2 and 3, progress in the understanding of this question could come from a study of the complete symmetry of the theory including the auxiliary fields $\varphi, \varphi^{\dagger}$ : the model contains zero modes in addition to the standard Goldstone $\pi$ coupled to $\bar{\chi} \chi$, clearly connected to the $\varphi \leftrightarrow \chi$ symmetry of the quenched action. In any case, a better insight into the nature of quenched QCD would be welcome.

It is a pleasure to thank J. Gasser, P. Hasenfratz, T. Jolicoeur, R. Lacaze, E. Laermann, H. Leutwyler, B. Petersson and J. Zinn-Justin for stimulating discussions. I am grateful to O. Napoly for his critical comments on the manuscript, and to D. Bunel for typing it.

\section{References}

[1] Li, L. F. and Pagels, H., Phys. Rev. Lett. 26 (1971) 1204.

[2] Gasser, J. and Leutwyler, H., Phys. Rep. C 87 (1982) 77.

[3] Gasser, J. and Leutwyler, H., Ann. Phys. 158 (1984) 142.

[4] Billoire, A., LaCAze, R., Marinari, E. and Morel, A., Nucl. Phys. B 251 [FS13] (1985) 581.

[5] Billoire, A., LACAze, R., Marinari, E. and Morel, A., Nucl. Phys. B 271 (1986) 461.

[6] Bowler, K. C., Chalmers, C. B., Kenway, R. D., Pawley, G. S., Roweth, D. and Wallace, D. J., presented by R. D. Kenway in the Proc. of Lattice Gauge Theory Workshop, B. Bunk, K. H. Mütter and K. Schilling Eds. (Wuppertal, Nov. 1985, Plenum Press, N.Y.) 1986.

[7] LaermanN, E., LANGHAMmer, F., SCHMitT, I. and Zerwas, P. W., Phys. Lett. B 173 (1986) 443.

[8] Morel, A., «The chiral limit in lattice QCD », in Proc. of the Lattice Gauge Theory Workshop,
B. Bunk, K. H. Mütter and K. Schilling Eds. (Wuppertal, Nov. 1985, Plenum Press, N.Y.) 1986.

[9] Jolicoeur, T., Kluberg-Stern, H., Lev, M., Morel, A. and Petersson, B., Nucl. Phys. B 235 (1984) 455 ; references to earlier works therein.

[10] Kluberg-Stern, H., Morel, A. and Petersson, B., Nucl. Phys. B 215 (1983) 527.

[11] Kogut, J., Stone, M., Wyld, H. W., Shenker, S. H., Shigemitsu, J. and SinClair, D. K., Nucl. Phys. B 225 (1983) 326.

[12] We thank LACAZE, R. for providing us with this analysis.

[13] LACAZE, R., «Meson spectrum and flavour symmetry restoration in $S U(2)$ quenched lattice QCD with staggered fermions », Saclay preprint PhT/86-093, to appear in Nuclear Physics B.

[14] We are indebted to GASSER, J., HASENFRATZ, P. and LEUTWYLER, H. for enlightening remarks on this subject. 